\title{
Transesophageal Echocardiography Guided Ligation of Right Pulmonary Artery to Left Atrial Fistula
}

${ }^{1}$ Sambhunath Das, ${ }^{2}$ Kalpna Irpachi, ${ }^{3} \mathrm{~V}$ Devagourou

\begin{abstract}
Right pulmonary artery to left atrial fistula is a rare congenital cardiac anomaly. A 23 years old man visited an ophthalmologist for complains of diplopia and diminished vision with cyanosis. Computerized tomography angiography diagnosed the presence of right pulmonary artery to left atrium fistula. Intraoperative use of transesophageal echocardiography confirmed the diagnosis and guided in real time for the successful ligation of fistula. Transesophageal echocardiography helped to perform the surgery without cardiopulmonary bypass.
\end{abstract}

Keywords: Right pulmonary artery to left atrial fistula, Diplopia, Transesophageal echocardiography, Computerized tomography angiography.

How to cite this article: Das S, Irpachi K, Devagourou V. Transesophageal Echocardiography Guided Ligation of Right Pulmonary Artery to Left Atrial Fistula. J Perioper Echocardiogr 2014;2(1):38-39.

Source of support: Nil

Conflict of interest: None

\section{CASE REPORTS}

Twenty-three-year old man, weighing $56 \mathrm{~kg}$, visited an ophthalmologist for complains of diplopia and diminished vision. Clinical examination detected history of palpitation, cyanosis and clubbing of fingers, and precordial murmur at pulmonary area. Subsequently, he was advised for cardiologist opinion. The transthoracic echocardiography (TTE) of the patient found situs solitus, levocardia, atrioventricular and ventriculoatrial concordance, 3 pulmonary veins draining to left atrium (LA), absence of atrial and ventricular septal defect, patent ductus arteriosus and aortopulmonary window. There was a right pulmonary artery (RPA) to LA shunt (right-to-left), dilated RPA of $30 \mathrm{~mm}$ diameter, small left pulmonary artery (LPA) of

\footnotetext{
${ }^{1,3}$ Additional Professor, ${ }^{2}$ Senior Resident

1,2Department of Cardiac Anesthesia, All India Institute of Medical Sciences, New Delhi, India

${ }^{3}$ Department of Cardiothoracic and Vascular Surgery, All India Institute of Medical Sciences, New Delhi, India
}

Corresponding Author: Sambhunath Das, Additional Professor, Department of Cardiac Anesthesia, Cardiothoracic Sciences Centre, All India Institute of Medical Sciences, Ansari Nagar, New Delhi-110029, India, Phone: +91-9868398105 e-mail: sambhunathds833@gmail.com
8 to $10 \mathrm{~mm}$. The LA was large and enlarged to $65 \mathrm{~mm}$ in size. Moderate LV dysfunction was present with ejection fraction of 30 to $35 \%$. ECG and Chest X-ray were of normal findings.

Computerized tomography (CT) angiography revealed a small jet of contrast traversing from proximal ascending RPA to LA causing aneurysmal dilatation of LA, and normal coronaries with right dominance. The patient was advised for the surgical ligation of fistula. Surgery was planned via median sternotomy with cardiopulmonary bypass (CPB) in standby. The patient received $6 \mathrm{mg}$ morphine and $25 \mathrm{mg}$ promethazine as premedication $1 \mathrm{hr}$ prior to surgery. In the operating room after establishing routine monitoring, anesthesia was induced and the trachea was intubated. Digital oxygen saturation was $84 \%$. The transesophageal echocardiography (TEE) examination using ultrasound machine (Philips iE33 model, Bothell, WA, USA) in upper midesophageal aortic short axis view with slight right ward rotation of the probe and at sector angle of $0^{\circ}$ revealed turbulent jet traversing from RPA to LA (Fig. 1). At midesophageal bicaval view, the RPA was visualized by the side of superior venacava (SVC). The fistula draining to LA was detected (Fig. 2). The TEE finding was discussed with cardiac surgeon and ligation of fistula without $\mathrm{CPB}$ was planned. The surgeon was able to dissect out the fistula and compress it, while TEE was used in real time to demonstrate the change in blood flow across the fistula.

The fistula was ligated and flow interruption across the fistula was verified on TEE imaging (Fig. 3). The oxygen saturation in the digital oximetry and arterial blood gas

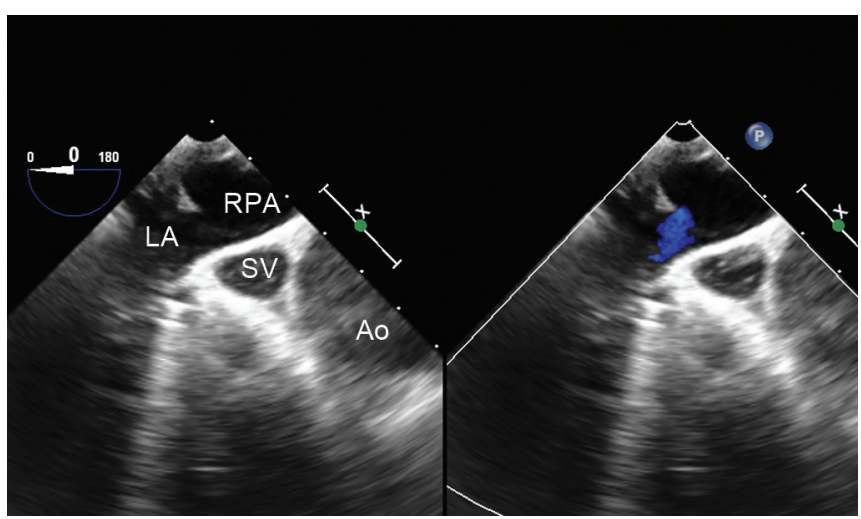

Fig. 1: Transesophageal echocardiographic image midesophageal aortic short-axis view showing right pulmonary artery to left atrial flow (SV: superior vena cava; LA: left atrium; RPA: right pulmonary artery, Ao: aorta) 


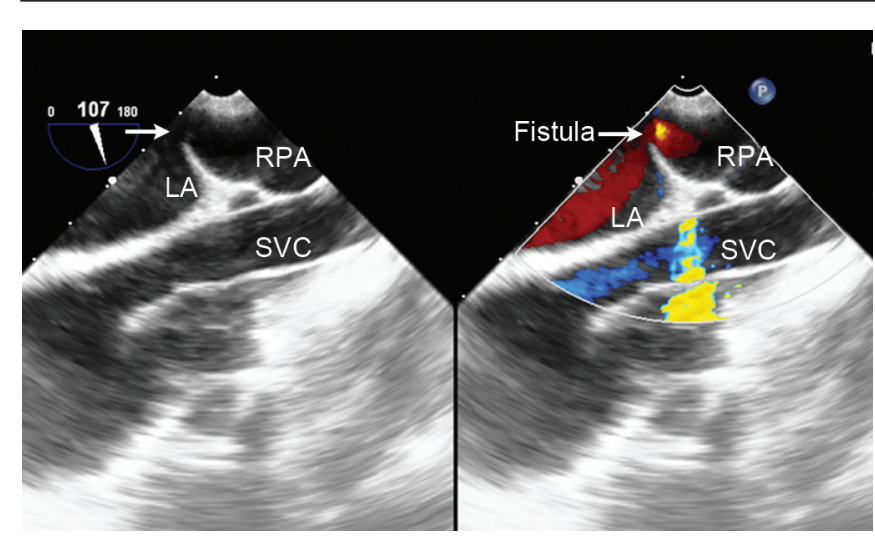

Fig. 2: Transesophageal echocardiographic image midesophageal bicaval view color Doppler showing right pulmonary artery to left atrial fistula (SVC: superior vena cava; LA: left atrium; RPA: right pulmonary artery)

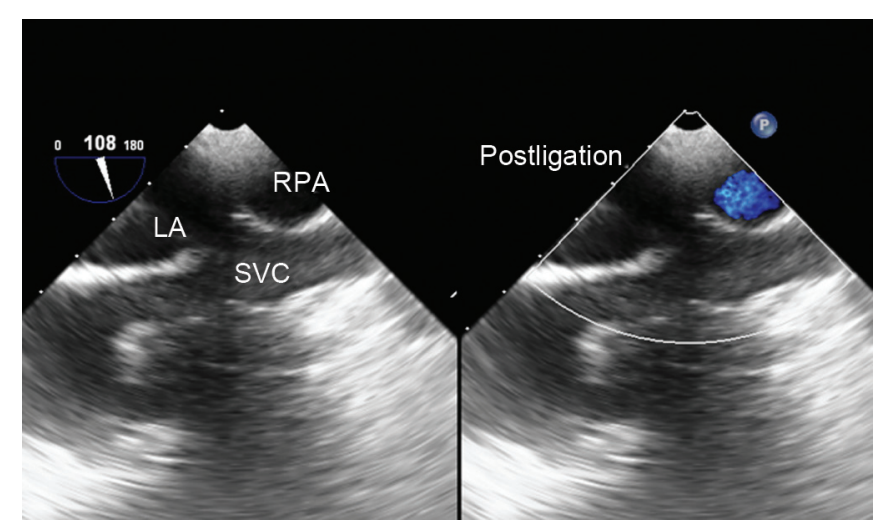

Fig. 3: Transesophageal echocardiographic image midesophageal bicaval view color Doppler showing no blood flow across right pulmonary artery to left atrium (SVC: superior vena cava; LA: left atrium; RPA: right pulmonary artery)

improved to $100 \%$. The trachea was extubated after 2 hours in the intensive care unit. The postoperative period was uneventful and he was discharged from hospital on 5th day.

\section{DISCUSSION}

Right pulmonary artery to left atrial fistula is a rare congenital cardiac anomaly. ${ }^{1}$ Definite mechanism of embryological development of this anomaly is not known. Few investigators have explained that the defect may result from a communication between RPA and a primitive pulmonary vein. Afterward the pulmonary vein becomes incorporated into the wall of the LA, resulting in a fistulous connection between the RPA and LA. ${ }^{2}$ The anatomical and angiographic findings in different case reports formulated four anatomical types of RPA to LA fistula. ${ }^{3}$

Type $I$ : Normal pulmonary venous connections.

Type II : The lower lobe branch of the right pulmonary artery communicates with an aneurysmally dilated right inferior pulmonary vein.

Type III : All pulmonary veins drain into the aneurysmal pouch between the RPA and the LA.
Type $I V$ : A very proximal pulmonary arteriovenous fistula joins the LA. The right pulmonary veins join the fistulous tract and the left pulmonary veins drain into the LA directly.

The usual presentation is central cyanosis, clubbing and cardiomegaly in childhood and adolescent age. Transthoracic echocardiography occasionally is unable to detect the RPA to LA fistula. ${ }^{4}$ Pulmonary angiography is the investigation to confirm the diagnosis. The treatment is surgery and transcatheter occlusion. ${ }^{5}$ The surgical ligation is most of the time performed with median sternotomy under $\mathrm{CPB}$ support. $^{6}$

In our case, the patient presented at 23 years of age with diplopia. The presence of cyanosis helped us to investigate and diagnose RPA to LA fistula. There might be systemic embolization through the fistula and might have caused stroke-like symptoms. In our case CPB was kept standby for the reasons that the identification of fistula might be difficult and accidental uncontrolled vessel injury would have been managed promptly. Intraoperative TEE was able to show the fistula connection which was also confirmed by surgical compression and decompression. Surgical ligation was performed in beating heart with simultaneous real time guidance of TEE without CPB. Transesophageal echocardiography also confirmed the successful ligation by the absence of flow in color Doppler. Hence intraoperative TEE is helpful tool to diagnose and guide the surgical intervention of RPA to LA fistula avoiding CPB and its related complications.

\section{REFERENCES}

1. Alexi-Meskishvili V, Dähnert I, Ovroutski S, Hetzer R. Right pulmonary artery-to-left atrium communication. A rare cause of systemic cyanosis. Tex Heart Inst J 2001;28:122-124.

2. Castleman B, Kibbee BU. Case records of the Massachusetts General Hospital: Case 45231. N Engl J Med 1959;260: 1180-1186.

3. Ohara H, Ito K, Kohguchi N, Ohkawa Y, Akasaka T, Takarada M, Aoki H, Ogata M, Nishibatake M, Fukatsu O, et al. Direct communication between the right pulmonary artery and the left atrium. A case report and review of literature. J Thorac Cardiovasc Surg 1979;77:742-747.

4. Veldtman G, Blackburn MEC, Parsons J. Images in cardiology. Congenital right pulmonary artery to left atrial fistula. Heart 1998;80:162.

5. Duke C, Alwi M. Transcatheter closure of direct communication between right pulmonary artery and left atrium using Amplatzer device. Heart 2003;89:1210.

6. Zeebregts CJ, Nijveld A, Lam J, Vanoort AM, Lacquet LK. Surgical treatment of a fistula between the right pulmonary artery and the left atrium: presentation of two cases and review of literature. Eur J Cardiothorac Surg 1997;11:1056-1061. 